\title{
CONTRIBUTION OF BATHYMETRIC MULTI-BEAM SONAR AND LASER SCANNERS IN 3D MODELING AND ESTIMATION OF SILTATION OF DAM BASIN IN MOROCCO
}

\author{
M. Bouziani ${ }^{1}$, F. Boucht ${ }^{2}$, F. Nouri $^{2}$ \\ ${ }^{1}$ School of Geomatic Sciences and Surveying Engineering, IAV Hassan II, BP 6202, Madinate Al Irfane, Rabat, Morocco - \\ (m.bouziani@iav.ac.ma) \\ ${ }^{2}$ National Agency for Land Administration, Cadastre and Cartography (ANCFCC), Morocco
}

KEY WORDS: 3D Modelling, Dam Monitoring, Siltation, Multi-beam Echo-Sounder, Laser Scanner, Point Cloud, Fusion

\begin{abstract}
:
In Morocco, the phenomenon of silting affects all the dams, where more than $75 \mathrm{Mm} 3$ of sediments are deposited every year at the bottom of the reservoirs. The aim of our study is to assess the contribution of the bathymetric multi-beam sonar as well as static and mobile laser scanners in the estimation of siltation of the basin of the Tanger-Med dam in the north of Morocco. The proposed methodology consists of performing and fusion of a bathymetric survey by multi-beam echo-sounder with terrestrial laser scanner surveys in static mode and mobile mode. The result of these surveys is used, for the calculation of the siltation volume and the inspection of the upstream facing of the main structure. Siltation calculation was carried out with comparison to a reference survey obtained by single beam sonar. The comparison between these two instruments showed that the multi-beam presents many advantages: high density of the cloud of points acquired and precision. The density allows providing a better description of the dam's bottom. The comparative study between the two types of scanners showed that static scanner offers a better accuracy. However, mobile scanner gives more accessibility of all parts of the dam. As a conclusion, the multi-beam echo-sounder with additional laser scanner data have many advantages in this study: volume calculation precision, reduction of the acquisition time and the enhancement of the acquired point cloud density. We also obtained a global $3 \mathrm{~d}$ modelling useful for the monitoring of the infrastructure.
\end{abstract}

\section{INTRODUCTION}

Currently, Morocco has more than 150 dams with a total capacity exceeding 18 billion $\mathrm{m} 3$, as part of a national strategy that envisages the resolution of water problems due to drought, limited water resources and semi-arid climatic conditions.

This strategy aims to secure the water supply and the economic and social development of the country, especially in the agricultural sector. These structures fulfil essential functions, namely: irrigation, drinking water reserves and flood control.

Consequently, monitoring of dams remains necessary in order to protect the structure against phenomena which may threaten its stability. Silting of dam reservoir is one phenomenon which reduces its storage capacity and threatens its safety. This sedimentation is due to the simultaneous effect of three actions: erosion, transport and sedimentation.

In Morocco, the phenomenon of silting affects all the dams, where more than $75 \mathrm{Mm} 3$ of sediments are deposited every year at the bottom of the reservoirs. The hydraulic development department, which is responsible for managing water resources and safeguarding hydraulic infrastructures, oversees the maintenance and regular control of these infrastructures. Volume determination must meet requirements in terms of precision, costs and completion time in order to consider and plan the next preventive actions, maintenance and sometimes removal of the silt volumes. Volume of sediment is usually determined using conventional methods that require a lot of time, effort and costs.

In recent years, many techniques are used to measure the underwater topographic data: single-beam echo-sounder, multibeam echo-sounder, and remote sensing methods like airborne Laser bathymetry and Satellite-derived bathymetry (IHO, 2018; Rossi et al., 2020, Yan et al., 2018). Some studies applied and assessed these technologies to different contexts for mapping and monitoring sediments and hydraulic infrastrctures (Rossi et al., 2020; IHO, 2018; Yan et al., 2018; Eleftherakis et al., 2014; Snellen et al., 2018; Gaida et al., 2020; Mohammadloo et al., 2020)

This work explores the advantages of using new techniques in Moroccan context: multi-beam echo-sounder, static and mobile laser scanner. The aim of our study is to assess the contribution of the bathymetric multi-beam sonar as well as static and mobile laser scanners in the estimation of siltation of the basin of the Tanger-Med dam in the north of Morocco. This work was carried out with the support of the IAV Hassan II, the directorate of hydraulic development, the Loukouss hydraulic basin agency and the following companies: Globetudes, Foresight and Geobat.

In the following sections, we will present the study area, the proposed methodology and the tools used for the experimentation. Then, we will give and discuss results. The article ends with the conclusion.

\footnotetext{
* Corresponding author
} 


\section{MATERIAL AND METHOD}

The proposed methodology consists of performing and fusion of a bathymetric survey by multi-beam echo-sounder with terrestrial laser scanner surveys in static mode and mobile mode. The result of these surveys is used, on the one hand, for the calculation of the siltation volume, and on the other hand, to inspect the upstream facing of the main structure.

Completing this job required three surveys. The first survey, bathymetric, is carried out using the Reason Seabat 7101 multibeam echo-sounder. The second survey is carried out with the leicaC10 static terrestrial laser scanner. The third survey is carried out using an MDL Dynascan mobile laser scanner.

The multi-beam echo-sounder has the advantage of covering a large area in a relatively short period of time. As for the static laser scanner, in addition to the significant gain in measurement time, it makes it possible to have a very dense, precise and reliable three-dimensional volume of information, which can be the subject of multiple processing. With regard to the mobile laser scanner, in addition to its ease of handling, it allows the scanning of large areas in a short time and the monitoring of the data collected in real time.

Siltation calculation was carried out with comparison to a reference survey obtained by single beam sonar. The comparison between these two instruments showed that the multi-beam presents many advantages. The most important of these advantages is the high density of the cloud of points acquired by the multi-beam. This density allows to provide a better description of the dam's bottom compared to the one provided by the single beam sonar.

\subsection{Study area}

The aim of our study is to assess the contribution of the bathymetric multi-beam sonar as well as static and mobile laser scanners in the estimation of siltation of the basin of the Tanger-Med dam in the north of Morocco (Figure 1).

The choice of Tanger-Med dam is justified by the availability of validation data and the need to conduct an inspection of the dam expressed by the partner, the hydraulic agency.

The dam of Tanger-Med, built in 2005 with an initial capacity of 25 Million cubic meters, with an average annual supply of 12 million cubic meters. The dam is supplied by Oued Rmel and its function is to cover the drinking and industrial water needs of Tanger-Med as well as the protection of the Tanger-Med port from flooding. The dam has a normal level surface of 123 hectars. The area of the watershed is $34 \mathrm{~km} 2$. The valley is roughly symmetrical with a bottom close to $45 \mathrm{~m}$ wide and fairly steep banks. The capacity of the dam has decreased over the years. This is explained by the siltation of the dam reservoir, the annual siltation rate is of 0.46 Million cubic meters per year.

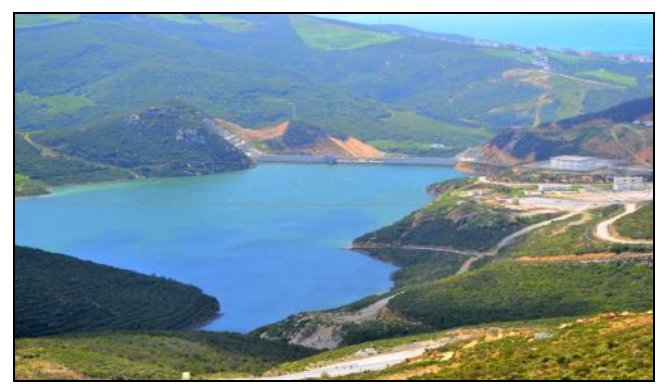

Figure 1. Tanger-Med dam in the north of Morocco.

\subsection{Methodology}

The proposed methodology consists of performing and fusion of a bathymetric survey by multi-beam echo-sounder with terrestrial laser scanner surveys in static mode and mobile mode. The result of these surveys is used, on the one hand, for the calculation of the siltation volume, and on the other hand, to inspect the upstream facing of the main structure.

Completing this job required three surveys. The first survey, bathymetric, is carried out using the Reason Seabat 7101 multibeam echo-sounder and the Minos SVP celerimeter. The second survey is carried out with the leicaC10 static terrestrial laser scanner. The third survey is carried out using an MDL Dynascan mobile laser scanner. The following figure presents the experimentation equipment.

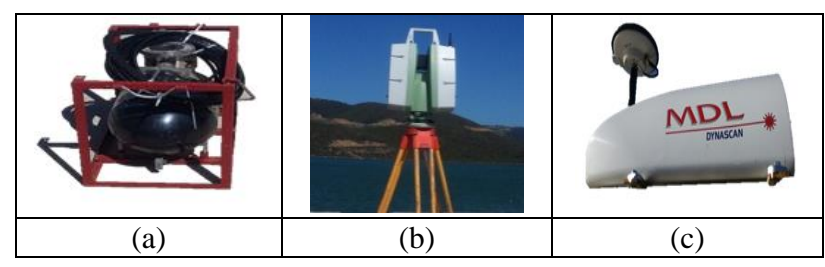

Figure 2. Equipment of experimentation: (a) Reason Seabat 7101 multi-beam echo-sounder, (b) Leica C10 TLS, (c) MDL Dynascan laser scanner

The multi-beam echo-sounder has the advantage of covering a large area in a relatively short period of time. As for the static laser scanner, in addition to the significant gain in measurement time, it makes it possible to have a very dense, precise and reliable three-dimensional volume of information, which can be the subject of multiple processing. With regard to the mobile laser scanner, in addition to its ease of handling, it allows the scanning of large areas in a short time and the monitoring of the data collected in real time.

The positioning equipment consists of a Leica GNSS receiver which occupies a fixed station, two Trimble GNSS receivers for heading calculation and another Leica GNSS receiver for positioning. The following figure shows the positioning GNSS receivers used in our study:
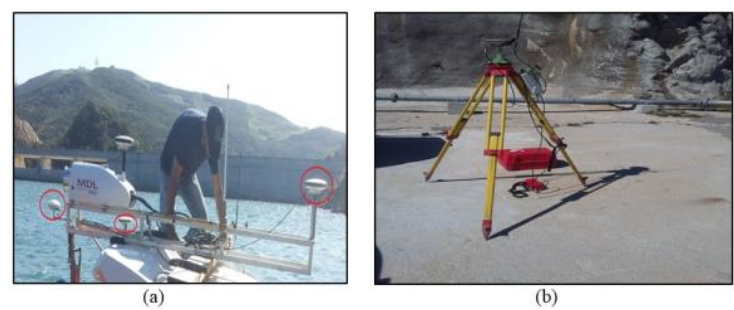

Figure 3. Positioning equipment: (a) GNSS receivers in RTK mode (b) GNSS receiver on the fixed station.

The operator interface allows the control of the system as well as the visualization of the data acquired in real time: raw signals, transverse bathymetric profile, bathymetry, sonar image, attitude, speed profile, surface speed and navigation. We used PDS2000 software which enables data acquisition, verification and editing, processing, volume calculation, 3D visualization and mapping. The attitude control unit used is the SMC IMU which provides the movement of the Galeon cruiser during data acquisition (Figure 4). 


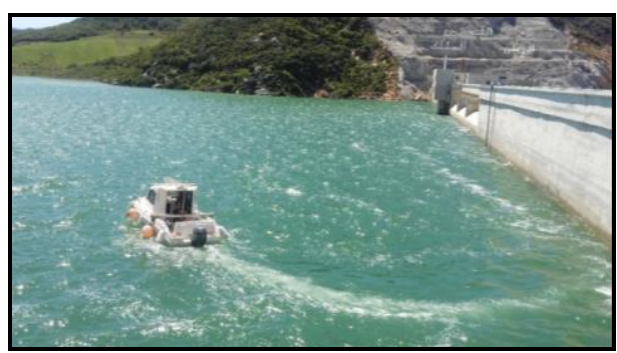

Figure 4. Galeon survey boat

To calculate the volume of the siltation and model the structure, we started by creating regular and homogeneous grids of the point clouds acquired. Subsequently, we assembled them. To do this, we imported all the clouds on the same interface. Since the surveys are made on the basis of the same tie points, viewing the assembled point cloud shows a good quality results. On the basis of these grids, we were able, on the one hand, to calculate the volume between the surfaces and, on the other hand, to model the entire scene.

The speed of the boat, as well as the range of the scanner, influences the resolution of the cloud obtained by multi-beam echo-sounder and by mobile laser scanner. For this, we created a regular and homogeneous grid with an interval of 1 meter covering the entire cloud. We choose this interval to facilitate the modelling. Kriging method is used for the interpolation of the point cloud.

For volume calculation, we imported and modelled the two point clouds: old (Reference) and recent (survey). Then, we created a TIN (triangulated irregular network) mesh which is based on the creation of triangles whose vertices are the closest points of the cloud. For the calculation of the siltation volume, we used Digital Terrain Models of the two surfaces (reference and survey) by the trapezoidal method which is based on calculations of surfaces integrals. The modelling is based on the creation of a mesh which best presents the studied surface. Once the surface was meshed, we smoothed it out to have an improved result for better visualization.

\section{RESULTS}

\subsection{Point clouds of the three surveys}

The figure 5, 6 and 7 present les three point clouds obtained.

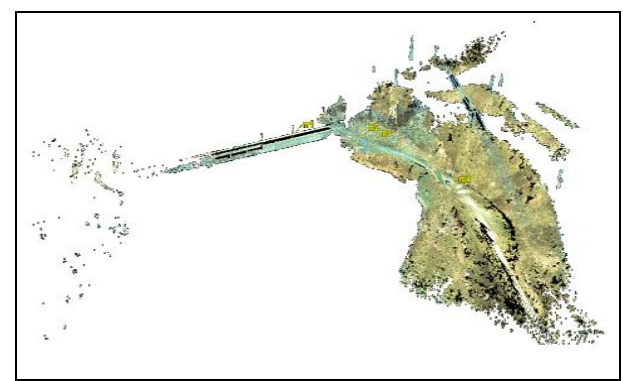

Figure 5. TLS point cloud

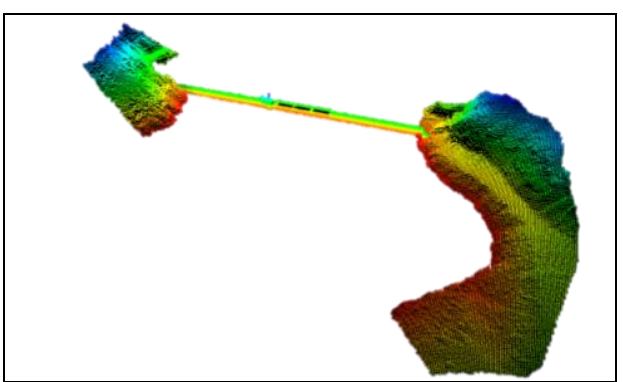

Figure 6. MDL Mobile scanner point cloud

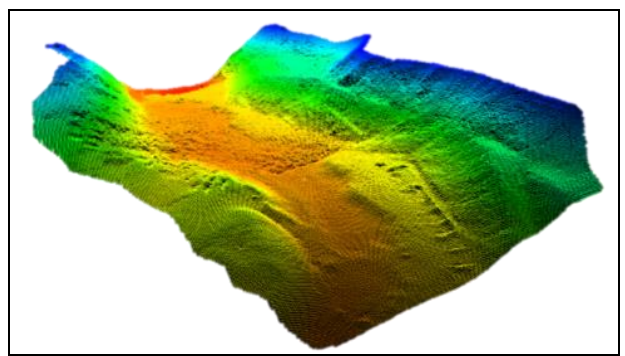

Figure 7. Point cloud obtained by the multi-beam echo-sounder

\subsection{Contributions of multi-beam bathymetry to map the dam reservoir}

Multi-beam bathymetry maps the bottom of the dam with high resolution. To assess the contribution of this technique, we carried out a comparison between the results obtained from the multi-beam echo-sounder during our study with the data provided by the hydraulic development department acquired by a single-beam echo-sounder (Raytheon), in the form of profiles separated from $25 \mathrm{~m}$ to $70 \mathrm{~m}$ and dense profiles near the bottom drain.

The coverage of the area to be surveyed by single-beam echosounder is done according to profiles while respecting a step predefined by the hydraulic development department which is of the order of $20 \mathrm{~m}$ to $30 \mathrm{~m}$ near the structure and 50 to 70 elsewhere, and $3 \mathrm{~m}$ between points of the same profile. For the multi-beam echo-sounder, it covers the entire holdback with a resolution of up to $30 \mathrm{~cm}$. The dense resolution of the multibeam echo-sounder allows better description of the bottom of the dam with a high resolution.

Accuracy is a very interesting benchmark for evaluating the effectiveness of each of these two echo-sounders. The determination of the depth of the basin depends on several errors such as : total error on the depth, error due to the speed, error due to the measurement of time, error due to heaving, error in draft, over-sinking and transducer squat.

The accuracy of the single-beam echo-sounder varies between $0.1 \%$ and $0.2 \%$ of the depth distance. The same level of accuracy is obtained for vertical measurement of the multi-beam echo-sounder. However, this precision is influenced by errors due to the refractive effect and the estimate of the roll angle which degrade the precision of the extreme beams.

The precision on the volume calculation does not only depend on the precision on the depth, but also on the density of the point cloud. The multi-beams provide a dense cloud which covers the entire basin area of the dam. Hence the interpolation performed during the mesh is precise. The volume calculation from multi-beam sonar is precise since it takes into account a maximum of details at the level of the surface on the basis of which this volume is calculated. 
The multi-beam echo-sounder measures in bands acquired at angles up to $210^{\circ}$ while the single-beam echo-sounder measures depths vertically, which limits its ability to acquire objects that are outside the vertical. In this regard, let us cite the example of the inclined limits of the banks of the dam which are not acquired when the single beam is used, on the other hand the multi-beam sonar ensures a complete presentation of the area and allows a better exploitation and interpretation of the results. In addition, the density of point clouds obtained by multi-beam makes it possible to use them not only in the calculation of siltation, but also for the monitoring of bottom emptying zones and the inspection of the submerged part of the structure. Many advantages are offered by the multi-beam echo-sounder but the major constraint lies in the cost of the service provided by this echo-sounder.

The acquisition of data on an area of 10 ha of the Tanger Med dam, using a multi-beam echo-sounder, took 1 hour for the installation of the equipment and the laying of the boat at the level of the dam using the crane, and 45 minutes for the data acquisition. If we project this duration to acquire the entire reservoir, which is 123 ha, we get 1 day. On the other hand, the single-beam survey of the same dam required 3 days to map the entire dam reservoir according to profiles spaced $20 \mathrm{~m}$ to $70 \mathrm{~m}$ apart.

\subsection{MDL and TLS Comparison}

To conduct the comparison between MDL mobile scanning and terrestrial laser scanning, we have chosen as the study area the common area between the two surveys as shown in the following figure.

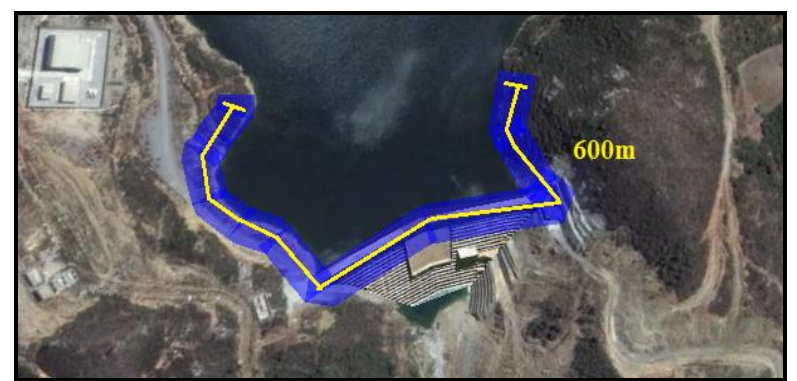

Figure 8. Study area for MDL and TLS comparison

The dimensions of the area are approximately $600 \mathrm{~m}$ in length and $50 \mathrm{~m}$ in width. The first survey is carried out by static scanner Leica $\mathrm{C} 10$ using 4 stations to cover the entire area. The acquisition by mobile scanner was done in a single pass using the MDL Dynascan scanner.

The analysis of the comparison results shows that the acquisition by static laser scanner is carried out with millimetre precision. The determination of the position by mobile scanner is carried out with centimetre precision. This precision varies with the range. The more the range increases, the more the precision degrades.

To assess the resolution and density of the two techniques, we performed a set of measurements on the two clouds, from a sample of a 1 meter square. We can conclude that the static scanner is efficient in terms of density, precision and resolution of the cloud. In fact, it offers a cloud six times denser than that of the mobile. However, the accuracy of the two scanners varies with range. This density enabled us to carry out an inspection at the level of the land part of the upstream facing of the main structure. Indeed, to carry out this inspection, we used the point cloud of the TLS scanner.
The total installation, acquisition and processing time is 5 hours for TLS and 3 hours for MMS. The mobile laser scanner offers a time saving of $40 \%$ than the static one. It achieves data acquisition in a single pass while the static scanner uses multiple stations to survey the entire scene in question. Acquisition by static scanner is limited by access difficulties in order to cover the entire dam. On the other hand, in terms of data processing, the static scanner requires processing of several steps more than the mobile.

When it comes to map only the structure or small dams, the use of the static laser scanner is recommended. However, if the purpose of the survey is to acquire the entire structure, in order to have a database that can be used for carrying out several measurements, the use of the mobile scanner is essential. In some dams, acquisition by static scanner is difficult or even impossible given the constraints of: visibility, scanner range and accessibility, in this case the use of a mobile scanner is required. Finally, we can conclude that in the choice of the technique to be used in the dams several criteria come into play and we must put forward our objectives and our financial capacities in order to be able to choose which of the techniques is more effective and efficient.

\subsection{Assessment of the fusion of point clouds}

To conduct the fusion assessment, we used methodology which is based on two surveys, bathymetric and lasergrammetric. This methodology consists in carrying out a bathymetric survey by multi-beam at a date $\mathrm{t} 1$ and waiting for the water level to be lower and carrying out another survey by a static laser scanner at a date $\mathrm{t} 2$ to constitute an overlap zone between the two surveys. Once the assembly between the two surveys is carried out, measurements of differences at the level of the overlapping zone are carried out to assess the reliability of the bathymetric data and to judge the continuity between the two surveys, lasergrammetric and bathymetric.

Another method consists to perform an extrapolation at the level of the two point clouds of the multi-beam sounder and laser scanners. The extrapolated points are assumed as if they were surveyed in the field. To check the reliability of the bathymetric data acquired by multi-beam echo-sounder we used data from the terrestrial laser scanner in static mode. To do this, we have chosen the transition area between the two data types as the control area. In fact, this area is located at the level of the upstream facing of the structure. This choice is justified by the fact that this zone firstly presents a regular shape easy for extrapolation and secondly the two types of data are acquired with a high resolution at this level. To carry out this extrapolation we used the Kriging method with an interval of 3 $\mathrm{cm}$ for the two point clouds. To quantify the difference that exists between the two clouds, we proceeded by calculating the distances between the closest points in the raw and extrapolated cloud. We obtained differences mainly vary as follows:

\begin{tabular}{lccc}
\hline & $\begin{array}{c}X \\
\text { Difference } \\
(\mathrm{cm})\end{array}$ & $\begin{array}{c}Y \\
\text { Difference } \\
(\mathrm{cm})\end{array}$ & $\begin{array}{c}Z \\
\text { Difference } \\
(\mathrm{cm})\end{array}$ \\
\hline Mean & 14 & 9 & 5 \\
\hline Standard deviation & 6 & 7 & 3 \\
\hline Minimum deviation & 10 & 2 & 2 \\
\hline Maximum deviation & 20 & 15 & 8 \\
\hline
\end{tabular}

Table 1. Fusion assessment results 
On the other hand, the results of the extrapolation prove that there are centimetre shifts between the two data, which can be explained by the fact that the two systems have two different orders of precision. The static laser scanner has millimetre precision while the multi-beam offers centimetre precision.

\subsection{Siltation calculation}

Siltation calculation was carried out with comparison to a reference survey obtained by single-beam echo-sounder. The following figures show the entire assembled point cloud and the modelling of the dam.

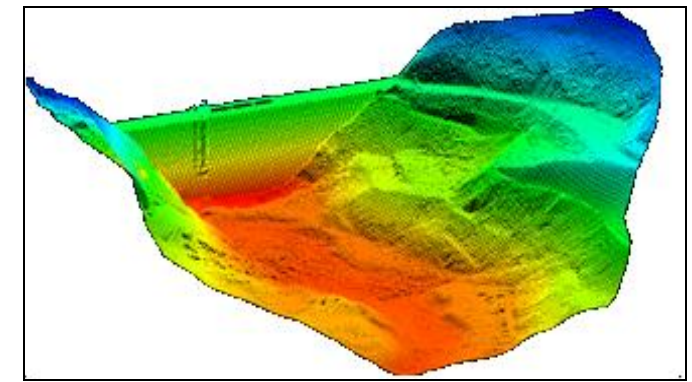

Figure 9. Assembly of point clouds on Cyclone software

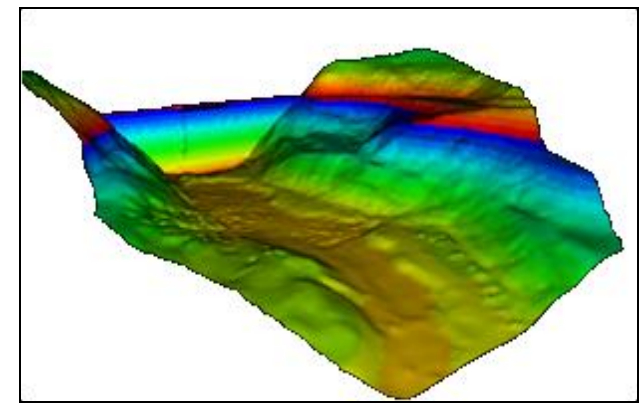

Figure 10. 3D model of the study area

\section{CONCLUSION}

In this study we conducted a comparative analysis between the single-beam and the multi-beam echo-sounders. From this analysis, we were able to conclude that the multi-beam echosounder has many advantages for dams modelling and monitoring, in terms of precision, acquisition time, completeness and density of the acquired cloud. We conducted also a comparative analysis between the laser scanner in static mode and in mobile mode. This analysis allowed us to conclude that the laser acquisition in static mode offers better precision compared to the acquisition in mobile mode, however the use of the static scanner is not always possible since some parts of the dams are not accessible so the mobile scanner is useful in these cases. Finally, we proposed a methodology for the fusion assessment of bathymetric and laser scanner data.

From this study, we were able to conclude that the multi-beam echo-sounder with additional laser scanner data have many advantages: volume calculation precision, reduction of the acquisition time and the enhancement of the acquired point cloud density. The fusion allows us to obtain a global 3D modelling useful for the monitoring of the infrastructure.

\section{REFERENCES}

Eleftherakis, D., Snellen, M., Amiri-Simkooei, A., Simons, D.G., Siemes, K, 2014. Observations regarding coarse sediment classification based on multi-beam echo-sounder's backscatter strength and depth residuals in Dutch rivers. The Journal of the Acoustical Society of America, 135 (6), 3305-3315.

Gaida, T.C., Mohammadloo, T.H., Snellen, M., Simons, D.G., 2020. Mapping the seabed and shallow subsurface with multifrequency multi-beam echo-sounders. Remote Sensing, 12 (1), 52.

International Hydrographic Organization (IHO), 2018. IHO Standards for Hydrographic Surveys, 5th ed. International Hydrographic Bureau: Monte Carlo, Monaco, 2018.

Rossi, L., Mammi I., Pelliccia, F., 2020. UAV-Derived Multispectral Bathymetry. Remote Sensing, 12 (23), 3897.

Snellen, M., Gaida, T.C., Koop, L., Alevizos, E., Simons, D.G., 2018. Performance of multi-beam echo-sounder backscatterbased classification for monitoring sediment distributions using multitemporal large-scale ocean datasets. IEEE Journal of Oceanic Engineering, 44 (1), 142-155.

Mohammadloo, T. H., Snellen, M., Simons, D. G., 2020. Assessing the Performance of the Multi-Beam Echo-Sounder Bathymetric Uncertainty Prediction Model. Applied Sciences, 10 (13), 4671.

Yan, Z. L., Qin, L. L., Wang, R., Li, J., Wang, X. M., Tang, X. L., An, R. D., 2018. The Application of a Multi-Beam EchoSounder in the Analysis of the Sedimentation Situation of a Large Reservoir after an Earthquake. Water, 10 (5), 557. 